\title{
Genetic diversity in tomato (Solanum lycopersicum L.) genotypes revealed by simple sequence repeats (SSR) markers
}

\author{
Ashish Kaushal ${ }^{1}$, Anita Singh ${ }^{1^{*}}$ and Anand Singh Jeena ${ }^{2}$ \\ ${ }^{1}$ Department of Vegetable Science, College of Agriculture, G.B. Pant University of Agriculture and Technology, \\ Pantnagar-263145(U.S. Nagar, Uttarakhand), INDIA \\ 2 Department of Genetics and plant Breeding, College of Agriculture, G.B. Pant University of Agriculture and \\ Technology, Pantnagar-263145(U.S. Nagar, Uttarakhand), INDIA \\ *Corresponding author. E-mail: anitasingh79@rediffmail.com
}

Received: July 22, 2016; Revised received: February 20, 2017; Accepted: May 3, 2017

\begin{abstract}
Twenty five tomato (Solanum lycopersicum L.) genotypes were subjected to genetic diversity analysis using twenty SSR markers. Out of 20 markers used, 14 SSRs were polymorphic and a total numbers of 22 SSR alleles were generated by 14 SSR markers, out of which 19 were polymorphic and 3 were monomorphic, with an average of 1.57 alleles per locus. The range of amplified products was $100-400 \mathrm{bp}$ approximately. Jaccard's similarity coefficient varied from 0.65 between germplasm EC519821 and CO-3 to a maximum of 1.0 between genotypes EC519769 and DARL-66, with an average value of 0.83 . Cluster analysis based on Jaccard's similarity coefficient using the unweighted pair-group method with arithmetic mean (UPGMA) revealed 2 distinct clusters, $A$ and B, comprising 1 and 24 genotypes respectively and at 75 and 78 per cent similarity, respectively. The genotypes which showed similar morphological and genetic trends were grouped more or less together in both these cases were a few. Cluster A comprised most diverse germplasm (EC519821)belongs to pimpinellifolium wild species with similarity coefficient $0.65 \%$ and differentiated with other cultivated species.Cherry Tomato and Cherry-2 were trends in similar cluster similar with approximately $96 \%$ similarity.SSR markers were able in in differentiating the genotypes based on morphologically and genotypically.However, the grouping of 25 genotypes were independently of geographic distribution. The genetic distance information found in this study might be helpful to breeder for planning among these genotypes.
\end{abstract}

Keywords: Genetic diversity, Jaccard's similarity coefficient, SSR, Tomato genotypes

\section{INTRODUCTION}

Tomato (Solanum lycopersicum L., formerly Lycopersicon esculentum Mill.) is the fourth most economically important crop in the world: after rice, wheat, and soybean and widely grown plants in Solanaceae family. Recently, tomato is a preeminent model system for genetic studies in plants having a small genome size (about $950 \mathrm{Mb} /$ haploid genome), availability of a wide range of high density molecular maps, DNA libraries (EST-expressed sequence tag and BAC-bacteria artificial chromosome) and microarrays (Barone et al.2008)

Diverse germplasm including specific genotype are the most valuable basic materials for crop breeder to meet the current and future needs. Sometimes, Morphological difference can not be interpreted to provide accurate estimates of genetic differences because morphological characters are highly influenced by the environments and $\mathrm{G} \times \mathrm{E}$ interaction (Shirasawa et al., 2013). In that situation, Molecular markers are powerful tools in the characterization and evaluation of genetic diversity within and between genetic populations (Russel et al., 1997). They have the advantage of providing thorough genome assessments' that are not influenced by the environmental factors, and directly reveals the polymorphism at DNA level (Ansari, 2015). Characterization of genotypes and varieties is mandatory for the purpose of registration with the competent authority and for granting plant breeder's rights under the criteria of Distinctness', Uniformity and stability(DUS).With the development of different molecular markers such as AFLPs (AmplifiedFragment Length Polymorphism), RAPD (Random Amplified Polymorphism) (Zhou et al.,2015), SSRs (Simple Sequence Repeats) are powerful tool for genetic diversity analysis and characterization of germplasm because of their reproducibility, co-dominant in nature and good genome coverage (Caguiat et al.,2014; Edris et al., 2014; Zhou et al., 2015)and SNPs (Single Nucleotide Polymorphism) are being used for diversity analysis, characterization, marker assisted selection and gene cloning (Corrado et al., 2013). Now a day, several molecular markers are developed, of which simple sequence repeats (SSRs) or microsatellites are the most widely used types. SSR markers have been successfully used in tomato for diversity analysis and 
characterization of tomato germplasms (Dhaliwal et al.,2011; El-Awady et al., 2012; Sanghani et al., 2014; Zhou et al., 2015). SSR marker is a PCR-based technique, which involves amplification of DNA fragments between adjacent and inversely oriented microsatellites. This technique uses microsatellites, usually $16-$ $25 \mathrm{bp}$ long, as primers. These primers can be di-, tri-, tetra- or penta-nucleotides (Zhang et al., 2014).

SSR marker fits worthy for a species like tomato, which has low level of variation as revealed by other markers (Bredemeijer et al., 2002). Improvement of crop depends on the magnitude of genetic variability in economic characters, therefore, the evaluation and utilization of genetic variability in desired direction becomes extremely important in any yield improvement program.

Thus, keeping the above fact in mind, the objective of this study was to assess the genetic diversity of tomato (S. lycopersicum L.) within the genotype using SSR markers.

\section{MATERIALS AND METHODS}

Plant materials: Out of 25 tomato ( $S$. lycopersicum L.) germplasm lines, 20 germplasm collected from core collection maintained at PCPGR (Pantnagar Centre for Plant Genetic Resources), Pantnagar, and 5 germplasm lines received from D.R.D.O., Pithoragarh, Uttarakhand. List of genotypes along with their source are given in Table 1.

SSR primers: Twenty SSR primer pairs were selected and synthesized from IDT (Integrated DNA Technologies, India). The detail of primer name, sequences, and corresponding annealing temperature and the amplified fragments are listed in Table 2.

Genomic DNA extraction: Healthy and mature leaf tissue (2 g) was ground in liquid nitrogen to a fine powder, and genomic DNA was extracted by using modified CTAB (hexa decyltrimethylammonium bromide) method (DOYLE and DOYLE, 1990) and quality of the DNA was estimated by separating it in $0.8 \%$ agarose gel stained with ethadium bromide.

DNA quantification and polymerase chain reaction: Purified DNA was quantified using GeneQuantUVspectrophotometer (GE Health Care Bio-sciences Ltd, U.K.) and diluted accordingly for further PCR analysis. The genotypes were screened for polymorphism with twenty microsatellite markers by slight modified method(Ollitrault et.al 2010). PCR amplification profile was carried out in $15 \mathrm{ul}$ volume containing $2 \mu \mathrm{L} 10 \mathrm{x}$ PCR reaction buffer, $25 \mathrm{mM} \mathrm{MgCl}_{2}, 1 \mathrm{mM}$ dNTP mix, 5 pmol of each (Forward \&Reverse) SSR primer and $20 \mathrm{ng}$ of template DNA, $1 \mathrm{U}$ of Taq DNA polymerase (3B Black Bio,Spain). PCR reactions were carried out in an Eppendorf mastercycler gradient thermocycler (Eppendorf, Hamburg, Germany) using the following temperature profile: initial step of $5 \mathrm{~min}$ at $94^{\circ} \mathrm{C}, 35$ cycles of $30 \mathrm{sec}$ at $94^{\circ} \mathrm{C}, 1 \mathrm{~min}$ at $50-63^{\circ} \mathrm{C}$
Table 1. List of genotypes and their sources used for diversity study.

\begin{tabular}{lll}
\hline S. No. & Genotypes & Source \\
\hline 1 & AC-576 & PCPGR, Pantnagar \\
2 & Sel-816-06 & PCPGR, Pantnagar \\
3 & ARTH-3 & PCPGR, Pantnagar \\
4 & EC-519821 & PCPGR, Pantnagar \\
5 & Cherry Sutton & PCPGR, Pantnagar \\
6 & EC-519812 & PCPGR, Pantnagar \\
7 & Cherry Tomato & PCPGR, Pantnagar \\
8 & EC-519977 & PCPGR, Pantnagar \\
9 & EC-519769 & PCPGR, Pantnagar \\
10 & CLN-2413 & PCPGR, Pantnagar \\
11 & PT-09-06 & PCPGR, Pantnagar \\
12 & Cherry-2 & PCPGR, Pantnagar \\
13 & EC-519818 & PCPGR, Pantnagar \\
14 & PT-19 & PCPGR, Pantnagar \\
15 & PT-8 & PCPGR, Pantnagar \\
16 & EC-519724 & PCPGR, Pantnagar \\
17 & DARL-67 & DRDO, Pithoragarh \\
18 & ArkaVikas & PCPGR, Pantnagar \\
19 & DARL-69 & DRDO, Pithoragarh \\
20 & DARL-68 & DRDO, Pithoragarh \\
21 & DARL-66 & DRDO, Pithoragarh \\
22 & DARL-62 & DRDO, Pithoragarh \\
23 & Shalimar & PCPGR, Pantnagar \\
24 & PantT-3 & PCPGR, Pantnagar \\
25 & CO-3 & PCPGR, Pantnagar \\
\hline
\end{tabular}

(depending upon the primer sequence) and $1 \mathrm{~min}$ at $72^{\circ} \mathrm{C}$ and a final step of $7 \mathrm{~min}$ at $72^{\circ} \mathrm{C}$. The amplification of PCR products were separated by using $3 \%$ agarose gel electrophoresis stained with ethidium bromide and documented by using UV-Pro gel documentation system.

Band scoring, estimation of diversity/ similarity and cluster analysis: The amplified bands were visually scored for the presence (1) or absence (0) in all the genotypes and the binary data were used for cluster statistical analysis by using NTSYS-pc version 2.11s software (Rohlf, 2002). The SIMQUAL program was used to calculate the Jaccard's similarity coefficients. Polymorphic information content (PIC) values were calculated for each SSR primers according to the formula given by (Smith et al.,1997).

$$
\mathrm{PIC}=1-\Sigma \mathrm{Pi}^{2}
$$

where $\mathrm{Pi}^{2}$ is the frequency of the $\mathrm{i}^{\text {th }}$ allele.

The similarity matrix value based on coefficient of similarity was used to generate dendrogram. Clustering was done by UPGMA using NTSYS-pc version 2.11s software (Rohlf, 2002).

\section{RESULTS AND DISCUSSION}

Microsatellite polymorphism analysis: SSR primers 
Ashish Kaushal et al. / J. Appl. \& Nat. Sci. 9 (2): 966 - 973 (2017)

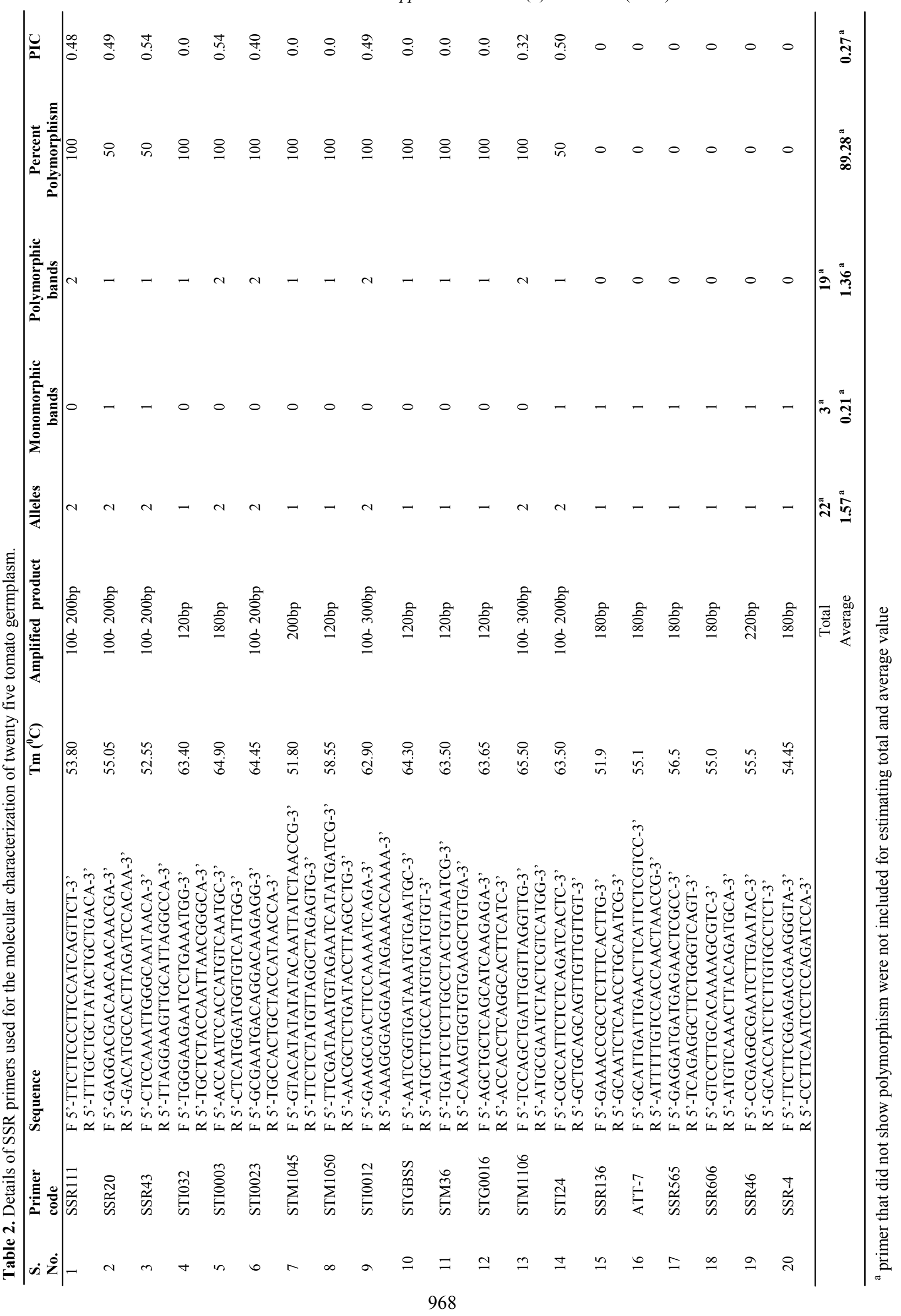


Table 3.Summary of SSR amplified fragments.

\begin{tabular}{lc}
\hline Total number of primer tested & 20 \\
Number of polymorphic primers & 14 \\
Total number of monomorphic primer & 06 \\
Total number of unique bands & 02 \\
Number of primer that gave unique bands & 02 \\
Total number of polymorphic bands & 244 \\
Total number of monomorphic bands & 125 \\
Total number of bands & 369 \\
Size range of amplified products (bp) & $100-300 \mathrm{bp}$ \\
Percent polymorphism & $50-100 \%$ \\
\hline
\end{tabular}

produced different numbers of DNA fragments, depending upon their simple sequence repeat motifs. The DNA was good in quality and quantity, free from proteins and RNA contamination of all 25 tomato genotypes used in this study. Out of twenty SSRs, six SSR markers viz., SSR136, ATT-7, SSR565, SSR606, SSR46 and SSR-4 amplified monomorphic banding patterns, hence were not considered for further analysis. Fourteen markers were thus used for final analysis based on scoreable amplified fragments. The number of SSR alleles scored, polymorphism detected, polymorphic information content observed for each 14 primers in 25 genotypes. DNA amplification profile for marker SSR111, STM1106, STI0012 and STI003 are presented in Fig.1. Fourteen SSR markers exhibited 369 bands in the twenty five tomato germplasm of which polymorphic and monomorphic bands were 244 and 125, respectively. The range of amplified products was $100-300 \mathrm{bp}$ approximately. Unique band were also observed by the primer namely STI0012 and STI0003 of approximate size of $210 \mathrm{bp}$ and170 bp, respectively. The primer STI0012 gave a unique band in CO-3 and primer STI0003 gave unique in germplasm DARL-69. A summary of SSR amplified fragments is presented in Table 2.

Similarity coefficient and cluster analysis: Data scored on twenty five tomato germplasm with 14 microsatellite (SSR) markers were used to generate Jaccard's similarity coefficient presented in Table 3 . In tomato germplasm, Jaccard's similarity coefficient varied from 0.65 between germplasm EC519821 and CO-3 to a maximum of 1.0 between varieties EC519769 and DARL-66, with an average value of 0.83 .UPGMA cluster analysis of genetic similarity matrix resulted in the dendrogram. All twenty five germplasm were demarcated at approximately 75 per cent similarity and further divided into two major clusters A and B (Fig.2) at 75 per cent similarity. The germplasm EC519821 was most diverse among all genotypes.

Molecular diversity was done to access the degree of genetic diversity or relatedness and to establish distinctiveness among the varieties. The PCR based molecu- lar marker system which SSRs were used for the study. The results are reported and discussed under the following heads. Fourteen SSR markers exhibited 369 bands in the twenty five tomato germplasm.

Benor et al. (2008)studied the genetic diversity of 39 determinate and indeterminate tomato lines and also reported the range of amplified products of 100-400 bp.Two unique bands were reported with two markers STI0012 and STI0003 in genotype CO-3 and DARL69 . These unique bands could have a number of potential applications including the determination of cultivar purity, efficient use and management of genetic resources collection and the establishment of property rights. In tomato germplasm, total numbers of 22 SSR alleles were generated by 14 SSR markers, out of which 19 were polymorphic and 3 were monomorphic, with an average of 1.57 alleles per locus. Al-Qadumiiet al (2012) reported 27 SSR alleles in land races of tomato grown by farmers with seventeen SSR markers. A range of polymorphism was observed from 50\% (SSR20, SSR43, and STI24) to 100\% (SSR111, STI032, STI0003, STI0023, STM1045, STM1050, STI0012, STGBSS, STM36, STG0016, and STM1106) with an average of 89.28\%.El-Awady et al. (2012), observed 38 amplified alleles in their study on Egyptian tomato cultivars, with 20 SSR markers out of which 23 alleles were polymorphic. The PIC value ranged from 0 to 0.54 with an average of 0.27 . The highest PIC (0.54) was observed with markers STI0003 and SSR 43. In a study on 39 tomato line the range of PIC from 0.05 to 0.60 were reported by Benor $e t$ al. (2008).

Similarity coefficient and cluster analysis: In tomato germplasm, Jaccard's similarity coefficient varied from 0.65 between germplasm EC519821 and $\mathrm{CO}-3$ to a maximum of 1.0 between varieties EC519769 and DARL-66, with an average value of 0.83. Similar results of were reported by Sanghani and mandivia(2013) in tomato. The germplasm DARL-66 and EC519769 was found 100\% similar to each other. The low range of similarity coefficient between any two genotypes indicated the presence of narrow genetic variability among the genotype studied.

The phylogenetic tree was constructed through NTSYSpc cluster analysis software using UPGMA (Un-weighted pair group method with arithmetic mean). UPGMA cluster analysis of genetic similarity matrix resulted in the dendrogram. All twenty five germplasm were demarcated at approximately 75 per cent similarity and further divided into two major clusters A and B (Fig.2) at 75 per cent similarity.

Cluster A comprised most diverse germplasm (EC519821). Cluster B was again bifurcated into sub clusters namely cluster $b_{1}$ and $b_{2}$ at $78 \%$ simi- 
Ashish Kaushal et al. / J. Appl. \& Nat. Sci. 9 (2): 966 - 973 (2017)

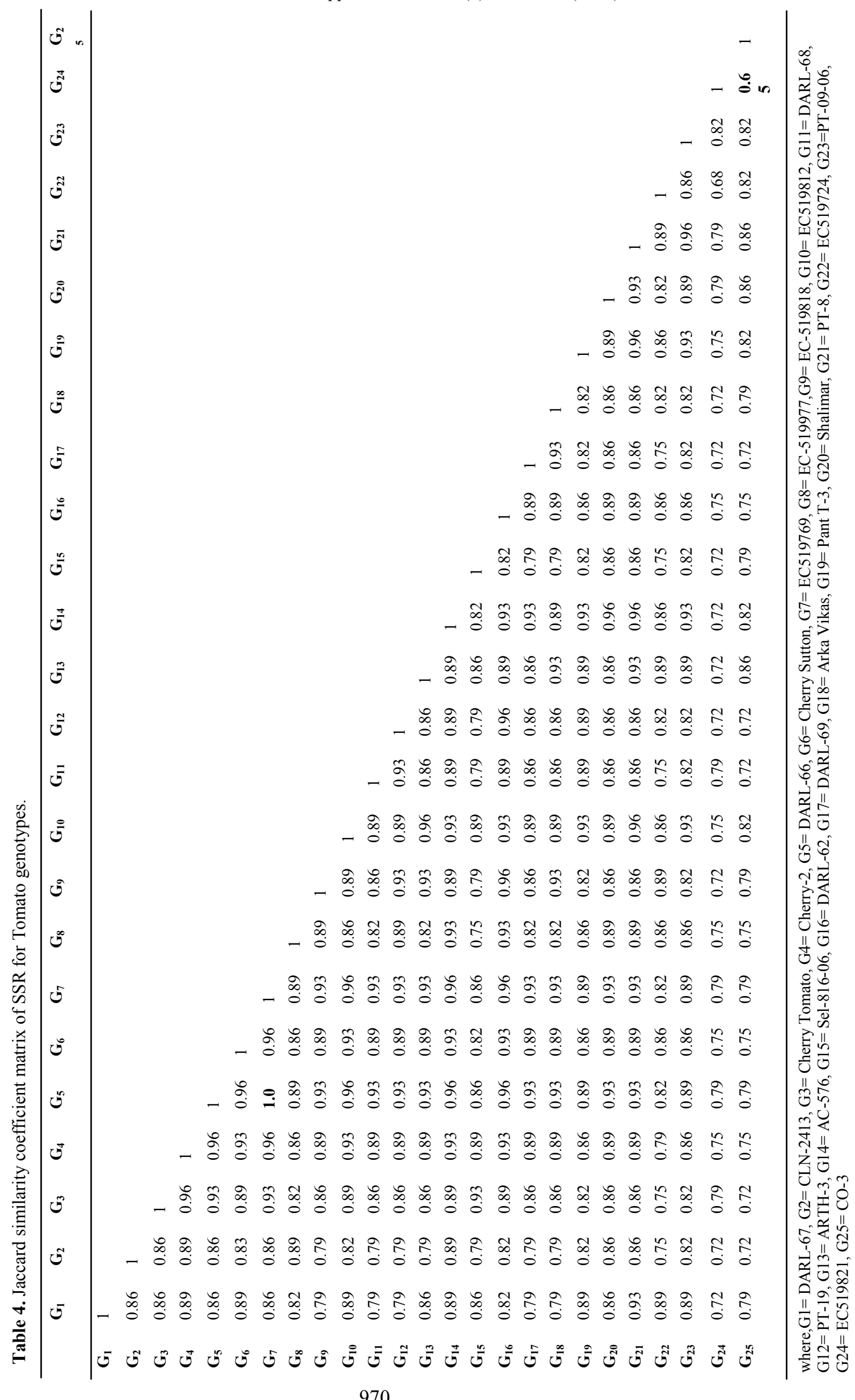


Ashish Kaushal et al. / J. Appl. \& Nat. Sci. 9 (2): 966 - 973 (2017)


Fig. 1. Amplification profile of 25 genotypes of tomato with SSR111, STM1106, STI0012 and STI003.(M=Marker $100 \mathrm{bp}$ ladder, line1-25(1=DARL-67, 2= CLN-2413, 3= Cherry Tomato, 4= Cheryy-2， 5= DARL-66, 6= Cherry Sutton, 7=EC519769, $8=E C-519977,9=E C-519818,10=E C 519812,11=D A R L-68,12=P T-19,13=A R T H-3,14=A C-576,15=$ Sel-816-06, 16= DARL-62, 17= DARL-69, 18= ArkaVikas, 19= Pant T-3, 20= Shalimar, 21 =PT-8, 22=EC519724, 23=PT-09-06, 24= EC519821, 25=CO-3)

larity. Cluster B1 consisted of 23 germplasm namely, DARL-67, EC519812, ARTH-3, Pant T-3, PT8, PT-09-06, EC-519724, Cherry Tomato, Cherry2, DARL-66, EC519769, Cherry Sutton, AC-576, Shalimar, EC519818, DARL-62, PT-19, DARL-68, DARL-69, ArkaVikas, CLN-2413, EC519977 and
Sel-816-06, while cluster $\mathrm{B}_{2}$ containing the single germplasm CO-3. Germplasm which are same by fruit size namely Cherry Tomato and Cherry-2 were trends in similar cluster similar with approximately $96 \%$ similarity. C0-3 which is mutant variety showed deviation from existing cluster was also 


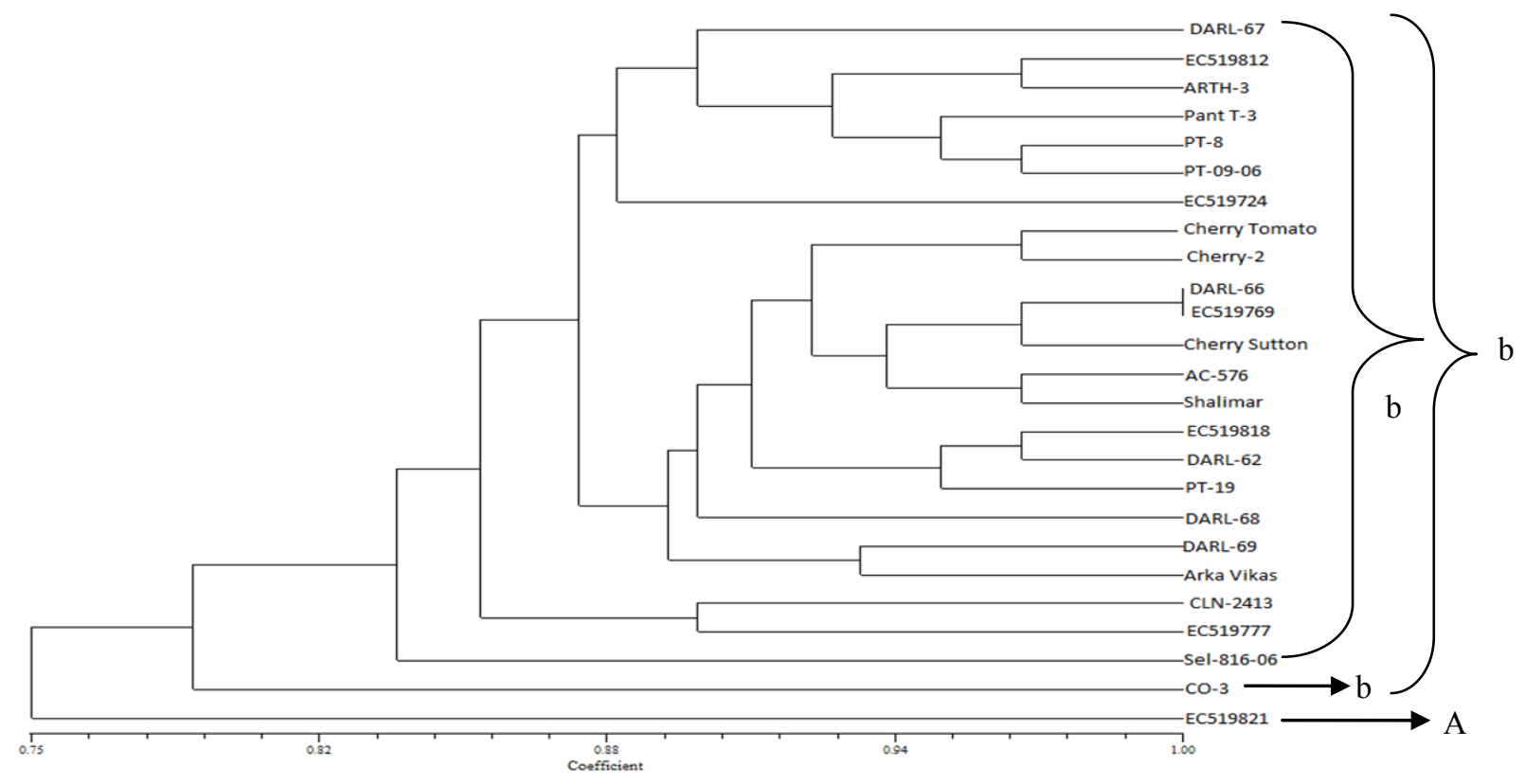

Fig.2. Dendrogram depicting the classification of the twenty five germplasm of tomato constructed using UPGMA method and based on SSR. The scale at the bottom is Jaccard's coefficient of similarity.

diverse with respect to their genetic makeup and morphological traits. The highest similarity Dendrograms showed a good fit between the graphical representation of distances and their original matrices. The germplasm which showed similar morphological and genetic trends were grouped more or less together in both these cases were a few. The very small fruited germplasm falls in most diverse cluster was EC519821 in cluster A, the present finding are consistent with the earlier report of Dhaliwal et al (2011). Cluster analysis revealed that the genotypes were not grouped according to the geographical distribution indicating that geographical distribution may not be criteria of genetic diversity in tomato. More or less they are grouped on the basis of horticultural and genetically makeup of genotypes.

\section{Conclusion}

It can be concluded that the SSR proved helpful for estimating the magnitude of genetic diversity in tomato at molecular level and also established genetic relatedness among genotypes studied.Cluster analysis revealed that the mutant variety $\mathrm{CO}-3$ is diverse with other genotypes studied among Solanum lycopersicum L. genotypes. The genotype EC915821, a very small fruited genotype of Solanum pimpinellifolium L. falls in most diverse cluster A.Hence SSR could differentiated diversity between $S$. lycopersicum L. and $S$. pimpinellifolium L. at species level. On the basis of banding pattern, SSR was effectively used for molecular characterization of tomato genotypes in the present study.
Therefore, there is need to support morphological diversity. It opens new perspectives towards conservation of tomato genetic resources and their further use in breeding programme.

\section{ACKNOWLEDGEMENTS}

Authors are thankful to the Director, Fxperiment Station, G. B. Pant. University of agriculture and Technology, Nodal officer, PCPGR and DRDO, Pithoragarhforproviding the germplasm and necessary facilities to conduct the present research.

\section{REFERENCES}

Al-Qadumii, L. W., Sadder,M. T. and Migdadi,H.(2012). Assessment of in silico BAC-based simple sequence repeat (SSR) marker development for tomato (Solanum lycopersicumL.).African Journal of Biotechnology, 11:13938-13946

Ansari, A., M.(2015). Molecular markers in vegetable improvement Horticultural Biotechnology Research.1(1):5 $-10$

Barone, A., Chiusano, M. L., Ercolano, M. R., Giuliano, G., Grandillo, S., \&Frusciante, L. (2008). Structural and Functional Genomics of Tomato. International Journal of Plant Genomics, 2008, 820274. http:// doi.org/10.1155/2008/820274

Benor,S.,Zhang, M. Y., Wang ,Z. F. and Zhang,H. S. ( 2008) Assessment of genetic variation in tomato (SolanumlycopersicumL.) inbred lines using SSR molecular markers Journal of Genetic Genomics, 35: 373379

Bredemeijer, G.M.M., Arens, P. and Wouters,D.( 1998) The use of semiautomated fluorescent microsatellite analysis for tomato cultivar identification. Theoretical and Ap- 
plied Genetics, 97:584-590

Caguiat X.G.I. and Hautea D.M. (2014).Genetic diversity analysis of eggplant (Solanum melongenaL.) and related wild species in the philippines using morphological and ssr markers SABRAO Journalof Breeding and Genetics, 46 (2) 183-201

Corrado, G. P., Piffanelli, M., Caramante, M., Coppola and Rao R. (2013): SNP genotyping reveals genetic diversity between cultivated landraces and contemporary varieties of tomato. BMC Genomics, 14: 835 doi:10.1186/1471-2164-14-835.

Dhaliwal, M. S., Singh, M., Singh, K. and Cheema, D.S. (2011).Genetic diversity analysis and DNA fingerprinting of elite genetic stock of tomato using SSR marker. Indian Journal Genetics, 71: 341- 348

Doyle ,J. J. and Doyle, J. L. (1990) Isolation of plant DNA from fresh tissues. Focus, 12: 13-15

Edris, S, S. Abo-Aba, M.M. Algandaby, A.M. Ramadan, N.O. Gadalla, M.A. AL-Kordy, J.S.M. Sabir, F.M. ELDomyatI, A.M. ALZhairy and A. Bahieldin (2014): Molecular characterization of tomato cultivars grown in Saudi Arabia and differing in earliness of fruit development as revealed by AFLP and ISSR. Life Science Journal, 11 (8): 602-612

El-Awady, M., El-Tarras, A.., A .E. and Hassan, M. M. (2012) Genetic diversity and DNA fingerprint study in tomato (Solanum lycopersicumL.) cultivars grown in Egypt using simple sequence repeats (SSR) markers. African Journal of Biotechnology, 1(1):16233-16240

Idrees M. and Irshad M.(2014).Molecular markers in plants for analysis of genetic diversity: a review. European academic research, (2) 1513-1540

Ollitrault, F., Terol, J., Pina J. A., Navarro L., Talon M. and Ollitrault, P.(2010).Development of SSR markers from Citrus clementina (Rutaceae) BAC end sequences and interspecific transferability in Citrus.American Journal of Botany, 97: 124-129

Rohlf, F.J.(2002).NT-SYS-pc: Numerical Taxonomy and Multivariate Analysis System. Version 2.1d. Exter Software: Setauket, NY.

Russel,J R., Fuller, J.D., Macaulay, M., Hatz, B. G., jahoo, A., Powell, W. and Waugh, R.(1997). Direct comparison of levels of genetic variation among barley accessions detected by RFLPs, AFLPs, SSRs and RAPDs. Theoretical and Applied Genetics, 95:714-22

Sanghani, A.O. and Mandavia, M. K. (2013). Characterization of tomato (Lycopersiconlycopersicum Mill.) genotypes through RAPDs, ISSRs, and SSRs marker. Indian Journal ofAgricuture Biochemistry, 26(2): 141-147

Shirasawa, K. and Hirakawa, H. (2013) DNA marker applications to molecular genetics and genomics in tomato. Breeding Science, 63(1):21-30. doi:10.1270/ jsbbs.63.21.

Smith ,J.S.C., Chin, E. C. L., Shu, H., Smith, O.S. and Wall, S. J.(1997).An evaluation of the utility of SSR loci as molecular markers in maize (Zea mays L.) comparision with data from RFLPs and pedigree. Theoretical and Applied Genetics, 95: 163-173

Zhang, S., Tang,C., Zhao, Q.,Li,J., Yang,L.,Qie,L.,Fan, X.,Li,L.,Zhang,N.,Zhao,M.,Liu,X.,Chai, Y.,Zhang, X.,Wang, H.,Li,Y.,Li, W.,Zhi, H.,Jia, G. and Diao,X. (2014) Development of highly polymorphic simple sequence repeat markers using genome-wide microsatellite variant analysis in Foxtail millet [Setariaitalica (L.) P. Beauv.]. BMC Genomics, 15:78. doi:10.1186/1471-2164-15-78.

Zhou, R., Wu, Z., Cao, X. and Jiang, F.L. (2015).Genetic diversity of cultivated and wild tomatoes revealed by morphological traits and SSR markers.Genet. Mol. Res., 14 (4): 13868-13879 